UDC 811.11-112'373

DOI: https://doi.org/10.18524/2307-4604.2019.2(43).186233

\author{
ÜBER PHONETISCHES MINIMUM IM DAF-UNTERRICHT \\ Nykyforenko I. \\ Dr. Phil., Nationale I.-I.-Metschnikow-Universität Odessa \\ ORCID ID: https.//orcid.org/0000-0003-2354-8581
}

Der Artikel befasst sich mit Fragen des phonetischen Minimums in Bezug auf die Kommunikationsfähigkeit mit dem Schwerpunkt „mündliche Kommunikation als globales Ziel des Fremdsprachenunterrichts“. Es handelt sich darum, welche phonetischen Inhalte Lernende beherrschen sollen, um bei der Entwicklung der Gesprächskompetenz die Sprache zu verstehen und selbst verstanden zu werden. Dafür braucht man je nach dem übergeordnetem Lehr- und Lernziel bestimmte phonetische Kompetenzen, Kenntnisse und Fertigkeiten. Im Unterricht selbst geht es allerdings nicht darum, das abstrakte, theoretische Phonemsystem zu vermitteln. Ein Lernziel ist es, die lautlichen Einheiten (Phoneme) des Deutschen und ihre Realisierungen in bestimmten, situativen Kontexten zu beherrschen. Dafür müssen Fertigkeiten der Perzeption (Hörwahrnehmung) und Produktion (Artikulation) entwickelt werden. Die Aussprache soll als integrierter Teil der Sprache verstanden werden. Die richtige Aussprache umfasst dabei sowohl den segmentalen Bereich der Vokale und Konsonanten, als auch den suprasegmentalen Bereich, d.h. die Intonation im Sinne der Einheit von Melodie, Rhythmus, Tempo, Pausenstellung und Akzentstruktur, was nämlich das phonetische Minimum bildet.

Schlüsselwörter: Deutsch als Fremdsprache, Gesprächskompetenz, phonetisches Minimum.

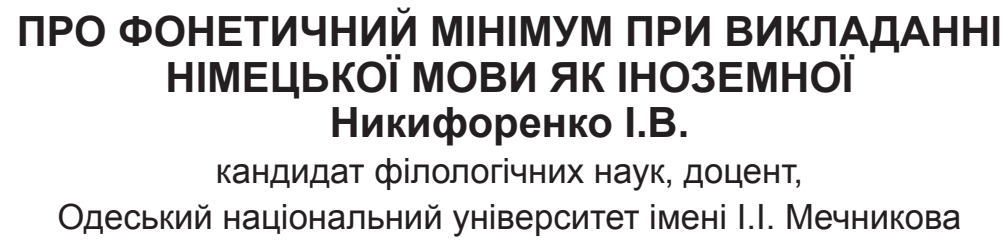

Стаття висвітлює питання вивчення фронетичного мінімуму у розвитку навичок усної комунікації як глобальної мети при вивченні іноземної мови. Між іншим розглядаються питання, пов язані з тим, якими фонетичними елементами повинні володіти ті, хто вивчає німецьку мову як іноземну, щоб цю мову розуміти та бути зрозумілими при опануванні комунікативної компетенції. Враховуючи основну мету, для цього потрібні певні фонетичні компетенції, знання та навички. При навчанні мова йде не лише про абстрактну теоретичну систему фонем, метою є оволодіння звуковими одиницями (фонемами) німецького мовлення та їх реалізацією в певних ситуативних контекстах, для чого потрібен розвиток навичок перцепції (сприйняття на слух) та продукції (артикуляції). Вимову треба розуміти як інтегровану частину мови. Правильна вимова охоплює при цьому не тільки сегментний рівень голосних та приголосних, а й суперсегментний рівень, тобто інтонацію як єдність мелодики, ритму, темпу, паузації та акцентної структури, що й становить фронетичний мінімум.

Ключові слова: німецька як іноземна, комунікативна компетенція, фонетичний мінімум.

\title{
ABOUT THE PHONETIC MINIMUM IN TEACHING GERMAN AS A FOREIGN LANGUAGE Nikiforenko I.V.
}

candidate of philological sciences, associate professor,

Odessa I.I. Mechnikov national university

The article addresses the issues of studying the phonetic minimum in the development of oral communication skills as a global goal in learning a foreign language. Among other things, the 
issues related to what phonetic elements should be learned when studying German as a foreign language in order to be understood and understand this language when mastering communicative competence, are being analyzed. Considering the main purpose, this requires certain phonetic competences, knowledge and skills. It is not just an abstract theoretical system of phonemes, the purpose of learning is to master the audio units (phonemes) of German speech and to realize them in certain situational contexts, which requires the development of perceptual (hearing) and production (articulation) skills. Pronunciation should be understood as an integrated part of the language. The correct pronunciation thus covers not only the segmental level of vowels and consonants, but also the super-segmental level, i.e. intonation as the unity of melody, rhythm, tempo, pausing and accent structure, which is the phonetic minimum.

Key words: German as a foreign language, communicative competence, phonetic minimum.

Die globale Informationsgesellschaft fordert heutzutage das Erlernen von Fremdsprachen zum beruflichen oder privaten Fortkommen. „Für die wirkliche Beherrschung einer Fremdsprache genügen allerdings Wortschatz und Grammatik der fremden Sprache nicht. Der Erfolg eines Gesprächs wird auch wesentlich durch Suprasegmentalia wie auch durch die Artikulation einzelner Phoneme bestimmt" (Hordula, 2007: 13).

Seit der Jahrhundertwende stellt der Erwerb mündlicher Kompetenz in verstärktem Maße das Hauptziel des DaF-Unterrichts dar. „Die in jener Zeit ihren Anfang nehmende kommunikative Fremdsprachendidaktik ist zugleich Träger und Ausdruck dieser Tendenz, indem sie das Lernen an Sprechhandlungen knüpft und damit die methodische Grundlage für einen Fremdsprachenunterricht schafft" (Hoffmann, 2017: 67).

In der Sprechwissenschaft und Phonetik sowie der Linguistik und Fremdsprachendidaktik besteht Klarheit darüber,

a) dass die Fertigkeiten Sprechen, Aussprache und Aussprachevermittlung wichtige und grundlegende Bestandteile des Fremdsprachenunterrichts sind;

b) dass phonetische Schwerpunkte in alle Bereiche des Sprachsystems (Lexik, Grammatik, Orthografie etc.) integriert und mit allen Fertigkeiten (Hören, Sprechen, Lesen, Schreiben) verbunden werden müssen;

c) dass die Grundlage für den Ausspracheunterricht und das Sprechen eine überregionale Standardaussprache ist, welche im gesamten deutschsprachigen Raum als Norm akzeptiert und verstanden wird;

d) dass die Lernenden darüber hinaus die situativen Varianten (z.B. Höflichkeitskonventionen, Redewendungen, Aussprache etc.) der Standardaussprache beherrschen und die nationalen/ regionalen Unterschiede (Dialekte, Akzente) verstehen und erkennen können sollen. Aus didaktischer Perspektive giltweiterhin derallgemeine Konsens, dass fürLernzielbestimmungen jeglicher Art im Fremdsprachenunterricht alle Sprachkenntnisse und Spracherfahrungen der Fremdsprachenlernenden relevant sind, miteinander in Beziehung stehen und interagieren (Malwitz, 2016: 16).

Unsere Forschungsfrage bilden Elemente phonetischen Minimums bei der Entwicklung der Mündlichkeit und Gesprächskompetenz im Fremdsprachenunterricht im Bereich der gesprochenen Sprache.

Ziel unserer Forschung ist es, eine Antwort auf die Frage zu liefern, auf welche Weise ein phonetisches Minimum aus theoretischem und praktischem Wissen zusammenzustellen ist und wie es in den Unterricht integriert werden kann. 
V. Malwitz ist der Meinung, dass das deutsche Phonemsystem ein erster wichtiger Ausgangspunkt für den Ausspracheunterricht ist. Im Unterricht selbst geht es allerdings nicht darum, das abstrakte, theoretische Phonemsystem zu vermitteln. Ein Lernziel ist es, die lautlichen Einheiten (Phoneme) des Deutschen und ihre Realisierungen in bestimmten, situativen Kontexten zu beherrschen. Dafür müssen Fertigkeiten der Perzeption (Hörwahrnehmung) und Produktion (Artikulation) entwickelt werden. Die richtige Aussprache umfasst dabei sowohl den segmentalen Bereich der Vokale und Konsonanten (im Bereich der Vokale: Vokallänge und Spannung, gerundete Vorderzungenvokale, Diphthonge, reduziertes [e] und Vokalneueinsatz; im Bereich der Konsonanten: Konsonantenspannung, Auslautverhärtung, Realisation der R-Laute, Hauchlaut [h], Ich-und Ach-Laut, progressive Assimilation), als auch den suprasegmentalen Bereich, d.h. die Intonation im Sinne der Einheit von Melodie, Rhythmus, Tempo, Pausenstellung und Akzentstruktur. Diese Schwerpunkte stellen das allgemeine „phonetische Minimum“ dar, um das Verstehen und Verstandenwerden in der Fremdsprache Deutsch zu garantieren (Malwitz, 2016: 17).

Im Fremdsprachenunterricht Deutsch hat sich der Stellenwert der Phonetik immer wieder verändert. Bis in die 1970er Jahre war eine „korrekte“ bzw. „normgerechte“ Aussprache erklärtes Lernziel. Mit der kommunikativen Orientierung wurde der Aussprache immer weniger Beachtung geschenkt, zu Beginn der 1990er Jahre enthielten DaF-Lehrwerke in der Regel keine Ausspracheübungen. „Dies ist eigentlich paradox, weil die elementare Basis für die mündliche Kommunikation vernachlässigt wurde. Gegenwärtig sieht die Situation wieder besser aus: Fast ausnahmslos alle Lehrwerke enthalten Ausspracheübungen, mehr oder weniger, gute oder weniger gute" (Hirschfeld, 2011: 10).

Der gemeinsame europäische Referenzrahmen (GeR) ist trotz seines Namens ein nominativer Text, der das Fremdsprachenlernen weltweit in bestimmte Bahnen lenkt. Er erschien zu einem Zeitpunkt, als der kommunikative Ansatz bereits fast ein Viertel Jahrhundert den dominierenden Rahmen der Fremdsprachendidaktik abgab (Rösler, 2016: 139-140). Es entstand aber die Frage, in wieweit im GeR auf die Spezifik von gesprochener Sprache und mündlichem Integrieren eingegangen wird, ob Differenzierung zwischen mündlicher und schriftlicher Produktion/Rezeption/Interaktion angelegt sei. Jetzt kann man festhalten, dass der GeR dadurch, dass er sich nicht auf die Unterscheidung von konzeptioneller Schriftlichkeit und Mündlichkeit einlässt, in dieser Hinsicht auch keinen Beitrag zu deren Bedeutung für das Fremdsprachenlernen liefert, dass er aber zumindest durch seinen Fokus auf Mündlichkeit Räume für eine differenzierende Beschäftigung mit Schriftlichkeit und Mündlichkeit bereitstellt (Verbitska, Nykyforenko, 2018: 42).

Nach dem Gemeinsamen europäischen Referenzrahmen für Sprachen zeigen sich die kommunikativen (d.h. linguistischen, pragmatischen und soziolinguistischen) Sprachkompetenzen eines Lernenden in verschiedenen kommunikativen Sprachaktivitäten, dazu gehören Rezeption, Produktion, Interaktion und Sprachmittlung (Dolmetschen und Übersetzung). Da der Handlungsaspekt eine wichtige Rolle spielt, spricht der Referenzrahmen von 
„kommunikativen Sprachtätigkeiten“, denen sich die „klassischen“ Fertigkeiten Hören, Sprechen, Lesen und Schreiben zuordnen lassen. Im Bereich der Mündlichkeit sind Hören und Sprechen die zentralen Sprachaktivitäten. Dabei müssen Lernende nach dem Referenzrahmen „über Erfahrungen mit der Ausführung einer Reihe von Handlungen verfügen“" (Hirschfeld, 2011: 12).

Diese phonetischen Fertigkeiten sind eng mit phonologischen und phonetischen Kompetenzen verbunden, die die Lernenden entwickeln müssen. Der Referenzrahmen nimmt hierzu ausführlich und in fachlich akzeptabler Form Stellung: „Phonologische Kompetenz [...] involviert Kenntnisse und Fertigkeiten der Wahrnehmung und der Produktion in Bezug auf:

1) die lautlichen Einheiten (Phoneme) der Sprache und ihre Realisierung in bestimmten Kontexten (Allophone);

2) die phonetischen Merkmale, die Phoneme voneinander unterscheiden (distinktive Merkmale,

z. B. stimmhaft, gerundet, nasal, plosiv);

3) die phonetische Zusammensetzung von Wörtern (Silbenstruktur, Phonemfolge, Wortakzent, Wortton);

4) Satzphonetik (Prosodie) - Satzakzent und Satzrhythmus - Intonation;

5) phonetische Reduktion - Vokalabschwächung - starke und schwache Formen - Assimilation - Elision" (GeR, Kap. 5.2.1.4: 117).

Eine leicht verständliche Aussprache setzt voraus, dass neue

Sprechbewegungen perfekt erlernt werden, d.h. sprechmotorische Abläufe - die auch in der Muttersprache nicht ohne Weiteres steuerbar sind - müssen bewusst gemacht und automatisiert werden. Diese Automatisierung ist etwas sehr Wichtiges, weil die Sprechbewegungen unbewusst korrekt ablaufen sollen; beim Sprechen konzentriert man sich mehr auf den Inhalt und die Kommunikationssituation als auf die Lippenrundung oder die Zungenbewegung. Esgenügtalso nicht, im Unterricht die Lautbildung zu erklären und auszuprobieren; wie bei jeder komplexen gymnastischen Übung ist ein ausgiebiges Training notwendig, bis alle Bewegungen perfekt ausgeführt werden. Dies gelingt nur, wenn Voreingenommenheiten bzw. Abneigungen gegenüber dem "fremden“ deutschen Klang überwunden werden, die Lernenden sich mit der eigenen Sprechweise in der fremden Sprache identifizieren und Sprechhemmungen abbauen (Hirschfeld, 2011: 14).

Nach U. Hirschfeld sind in der Interaktion, also im Gespräch oder in der Diskussion, die genannten rezeptiven und produktiven Fertigkeiten grundlegend für eine adäquate Verständigung und den gewünschten Gesprächsablauf. „Dazu gehört auch die Sensibilisierung für nonverbale und paraverbale Elemente, also für Stimmklang, Mimik und Gestik. Darüber hinaus müssen auch in einer Fremdsprache und in einer anderen Gesprächskultur Fähigkeiten zur Empathie sowie zur Perspektivenübernahme, zur Situations- und Partnereinschätzung entwickelt werden " (Hirschfeld, 2011: 14).

Bei der Vermittlung phonetischer Grundlagen muss also auf die Funktion, die Perzeption und Rezeption, sowie die Produktion der gesprochenen Sprache Bezug genommen werden. Inwieweit die Aussprachevermittlung in den Unterricht integriert wird, hängt zum einen vom Fachwissen der Lehrenden ab, 
zum anderen von der Priorität und damit der Zeit, die innerhalb des Unterrichts eingeräumt wird. Diese kann aber effizient genutzt werden, wenn Lehrende ein „phonetisches Minimum“ für ihre Studierenden ermitteln. Dabei ist es wichtig, dass die Ausgangsfertigkeiten und Lernziele ihrer Zielgruppe berücksichtigen, phonetische Lerninhalte gezielt auswählen und sie systematisch unterrichten (Malwitz, 2016: 20).

Das Lernziel der phonetischen Verständlichkeit erweist sich als wichtige und bislang praktikabelste Grundlage, um sowohl segmentale als auch suprasegmentale Inhalte nach ihrer Relevanz im kommunikativen Kontext abzustufen. Allerdings sind weitere Forschungen zum schrittweisen Erwerb segmentaler und suprasegmentaler Einheiten in der Fremdsprache Deutsch notwendig, um den Zusammenhang der angeführten Ansätze mit Spracherwerbbesonderheiten genauer zu untersuchen und die Kriterien für eine phonologisch-phonetische Progression in der Aussprachevermittlung abzusichern (Bose, Hannken, Hirschfeld, Neuber (Hrsg.), 2017: 160).

Es versteht sich in diesem Zusammenhang von selbst, dass im Unterricht die praktische Seite der Phonetik im Vordergrund stehen sollte. Deshalb ist darauf zu achten, dass man sich auf ein Minimum an Theorie beschränkt. Dabei ist es wichtig, die Ausgangsfertigkeiten und Lernziele der Zielgruppe zu berücksichtigen, phonetische Lehrinhalte gezielt auszuwählen und sie systematisch zu unterrichten.

\section{Literatur}

Bose I., Hannken K., Hirschfeld U., Neuber B. (Hrsg.) Forschung und Didaktik der Sprechwissenschaft: Aktuelle Beiträge 2017. 145-165.

Europarat (2001). Rat für kulturelle Zusammenarbeit: Gemeinsamer europäischer Referenzrahmen für Sprachen: lernen, lehren, beurteilen. Langenscheidt, online unter: http:// www.goethe.de/z/50/commeuro/i3.htm

Hirschfeld U. Phonetik im Kontext mündlicher Fertigkeiten. Babylonia 02/11, 10-17. 2011. URL:http://babylonia.ch/fileadmin/user_upload/documents/2011-2/Baby2011_2hirschfeld.pdf

Hirschfeld U. Aussprache in ihrer Vielfalt erleben: Lehr- und Lernziele. Fremdsprache Deutsch. Zeitschrift für die Praxis des Deutschunterrichts, 2016. 55, 10-15.

Hoffmann S. Bewusstheit und ihre Ermittlung in der Unterrichtskommunikation Bestandsanalyse und Perspektiven. Deutsch als Fremdsprache. Zeitschrift zur Theorie und Praxis des Faches DaF, 2, 2017. 67-74.

HordulaC. UntersuchungenzusegmentalenundsuprasegmentalenAusspracheabweichungen, 2007. 13-15.

Lüger H.-H. Authentische Mündlichkeit im fremdsprachlichen Unterricht. Mediale Varietäten. Gesprochene und geschriebene Sprache und ihre fremdsprachendidaktischen Potenziale. Beiträge zur Fremdsprachenvermittlung, 15, 2009. 15-16.

Malwitz V. Gibt es ein phonetisches Minimum? Fremdsprache Deutsch. Zeitschrift für die Praxis des Deutschunterrichts, 55, 2016. 16-20.

Nykyforenko I., Verbitska T. Diskurspartikeln bei der Entwicklung der digitalen Kommunikationsfähigkeit. Записки з романо-германської філології, 1 (40), 2018. 41-47. DOI: https://doi.org/10.18524/2307-4604.2018.1(40).137057

Rösler D. Nähe und Distanz zur Mündlichkeit in der fremdsprachendidaktischen Diskussion. Deutsch als Fremdsprache. Zeitschrift zur Theorie und Praxis des Faches DaF, 3, 2016. 139-140.

\section{References}

Bose, I., Hannken, K., Hirschfeld, U., Neuber, B. (Hrsg.), (2017). Forschung und Didaktik der Sprechwissenschaft: Aktuelle Beiträge, 145-165. 
Europarat (2001). Rat für kulturelle Zusammenarbeit: Gemeinsamer europäischer Referenzrahmen für Sprachen: lernen, lehren, beurteilen. Langenscheidt, online unter: http:// www.goethe.de/z/50/commeuro/i3.htm

Hirschfeld, U. (2011). Phonetik im Kontext mündlicher Fertigkeiten. Babylonia 02/11, 10-17. http://babylonia.ch/fileadmin/user_upload/documents/2011-2/Baby2011_2hirschfeld.pdf

Hirschfeld, U. (2016). Aussprache in ihrer Vielfalt erleben: Lehr- und Lernziele. Fremdsprache Deutsch. Zeitschrift für die Praxis des Deutschunterrichts, 55, 10-15.

Hoffmann, S. (2017). Bewusstheit und ihre Ermittlung in der Unterrichtskommunikation Bestandsanalyse und Perspektiven. Deutsch als Fremdsprache. Zeitschrift zur Theorie und Praxis des Faches DaF, 2, 67-74.

Hordula, C. (2007). Untersuchungen zu segmentalen und suprasegmentalen Ausspracheabweichungen, 13-15.

Lüger, H.-H. (2009). Authentische Mündlichkeit im fremdsprachlichen Unterricht. Mediale Varietäten. Gesprochene und geschriebene Sprache und ihre fremdsprachendidaktischen Potenziale. Beiträge zur Fremdsprachenvermittlung, 15, 15-16.

Malwitz, V. (2016). Gibt es ein phonetisches Minimum? Fremdsprache Deutsch. Zeitschrift für die Praxis des Deutschunterrichts, 55, 16-20.

Nykyforenko, I., Verbitska, T. (2018). Diskurspartikeln bei der Entwicklung der digitalen Kommunikationsfähigkeit. Zapysky z romano-hermanskoi filolohii, 1 (40), 41-47. DOI: https://doi. org/10.18524/2307-4604.2018.1(40).137057

Rösler, D. (2016). Nähe und Distanz zur Mündlichkeit in der fremdsprachendidaktischen Diskussion. Deutsch als Fremdsprache. Zeitschrift zur Theorie und Praxis des Faches DaF, 3, 139-140.

Стаття надійшла до редакції 3.10.2019 року 